\title{
Numerical analysis of passive seismic emission tomography using oscillation caused by multiphase flow method
}

\author{
Akitomo WATANABE ${ }^{1}$, Hitoshi MIKADA ${ }^{1}$ and Junichi TAKEKAWA ${ }^{1}$ \\ ${ }^{1}$ Dept. of Civil and Earth Res. Eng., Kyoto University
}

\begin{abstract}
In recent years, development of a monitoring technique (Passive Seismic Emission Tomography method: PSET) using vibration generated in the reservoir has been widely progressed. However, monitoring with PSET only estimates the extent of the reservoir. Moreover, its theoretical knowledge is lacking at present. On the other hand, we proposed a method to monitor underground from vibration caused by fluid flow inside the reservoir. We are conducting research based on the hypothesis that the difference in the fluid in the reservoir is reflected to the seismic wave generated in the reservoir. In the previous research, we showed that it is possible to visualize the reservoir area by measuring the seismic wave caused by the two-phased oil and water flow for a long time. In this study, we calculate seismic waves using the Green's function occurring with water-oil two-phase flow and gas-oil two-phase flow. As a result, the vibration caused by the flow of the fluid not only indicates the flowing area but also information on the properties of the fluid. It is suggested that this result may be effective as a method to monitor the change of the fluid properties in the reservoir associated with oil production and enhanced oil recovery method.
\end{abstract}

\section{INTRODUCTION}

In the field of oil development, the Passive Seismic Emission Tomography method (PSET), which is a monitoring method using the vibration generated in the reservoir, has attracted attention. For example, Ted (2016) succeeded in estimating the position of the production well using only the vibration generated by the fluid flowing through the production well, in a noisy environment near the roadway ${ }^{1)}$. This shows the possibility of obtaining significant information among the vibrations caused by fluid flow buried in noise. Erokhin (2014) also shows an example of PSET at the oil development site in Siberia ${ }^{2)}$. He not only can estimate the position where hydraulic fracture occurred by monitoring micro earthquakes, but also suggests the possibility of analyzing the fluid flow region. However, although there are some examples, theoretical consideration of vibration due to fluid flow is lacking. So, the relationship between observed seismic data and fluid information for example velocity, viscosity and fracture network in the subsurface has not been well understood yet. Therefore, in this study, we investigated what type of stress disturbance is caused by fluid flow using numerical simulation using the following procedures (Figure 1).

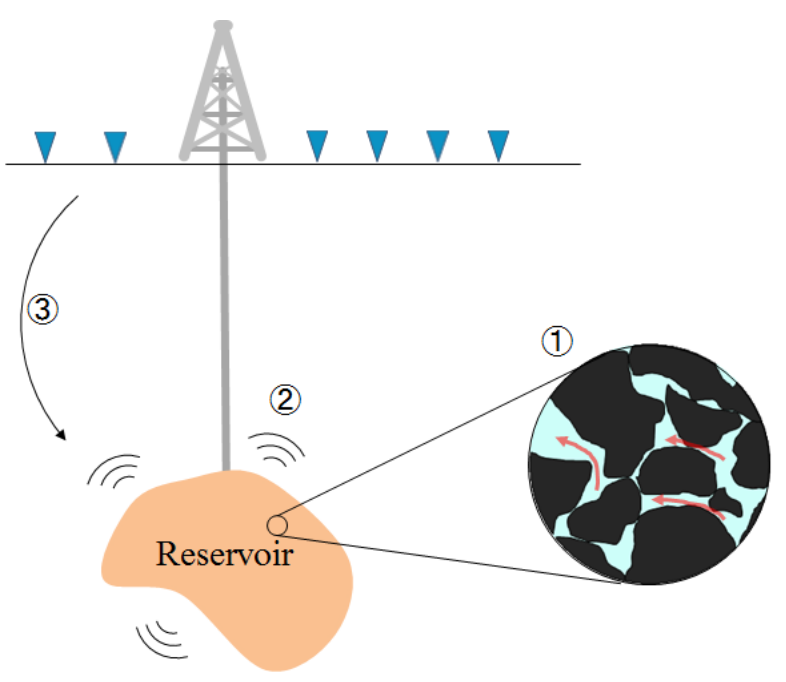

Figure 1 research flow

(1) calculate fluid flow in reservoir and vibration.

(2)propagate seismic wave caused by fluid flow to surface receivers.

(3)estimate reservoir from received waveform.

\section{METHOD}

To simulate fluid flow, we used the lattice Boltzmann method (LBM). The LBM is superior to ease of parallelization, simplicity of complicated boundary setting, and so on. In this method, we define the density distribution function of virtual microfluidic particles and numerically solve the evolution equation of the distribution function (Figure 2). Therefore, this method does not describe 
macroscopic fluid motion itself. However, it is shown that the Navier-Stokes equations can be derived from the equations used in LBM. So, its consistency is guaranteed.

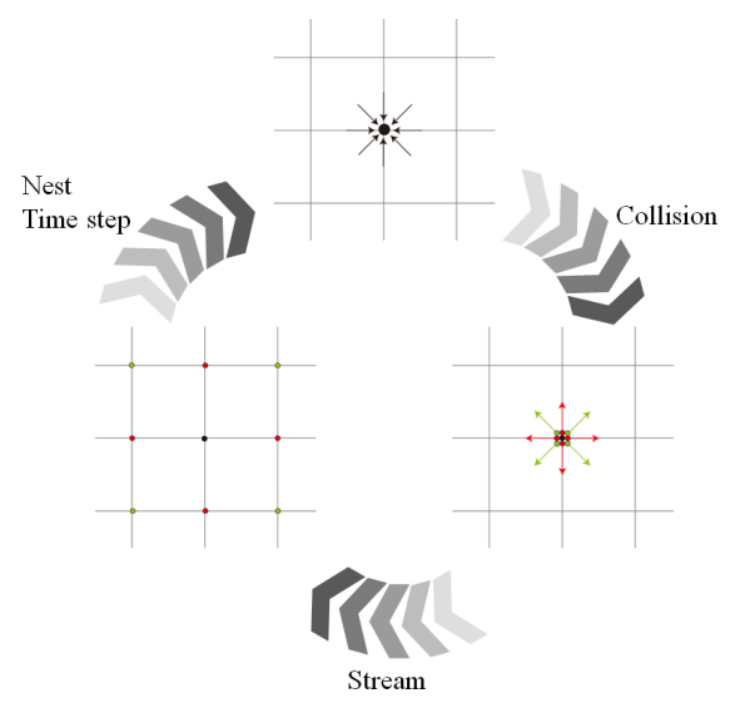

Figure 2 Lattice Boltzmann method

We simulate low density difference two-phase fluid flow and high density difference two-phase fluid flow. In the both fluid simulation, the two-dimensional nine-directional (D2Q9) lattice Bhatnager-Gross-Krook model (LBGK) is adopted. In small density difference two-phase fluid flow, we adopt R-K model ${ }^{3)}$ for multi-phase flow. On the other hands, in big density difference two-phase fluid flow, we adopt special LBM method developed by Inamuro (2004) $)^{4)}$. This method improved Swift-Osborne-Yeomans model ${ }^{5)}$.

In this paper, we use the Green function to calculate seismic waves which are caused by oil droplet or gas bubble through pore-throat. When external force is shown by $f(x, t)$, wave equation is given as:

$$
\frac{1}{\alpha^{2}} \frac{\partial^{2} \varphi}{\partial t^{2}}=\nabla^{2} \varphi(x, t)+f(x, t)
$$

Using the Green function $G\left(x, t ; x_{0}, t_{0}\right)$, that wave equation's solution is given as:

$$
\varphi(x, t)=\int_{-\infty}^{+\infty} d t_{0} \int_{V} G\left(x, t ; x_{0}, t_{0}\right) f\left(x_{0}, t_{0}\right) d V_{0}
$$

If we calculate the Green function only once, arbitrary responses can be obtained by the superposition.

After the propagation waveform and calculate received waveform at surface receivers, we calculate cross correlation each received waveform to estimate the hypocenter i.e. received fluid flowing area. Taking cross correlation, we gain two merits. Firstly, we can collect the long-term feeble oscillation energy. Unless the energy of the received waveform over a long period is aggregated, it is impossible to separate the vibration caused by fluid flowing from the received waveform buried in random noise. Secondly, we can know that which receiver is near hypocenter, and difference of distance between two receivers from hypocenter. Using this information, we can estimate fluid flowing area.

\section{MODEL}

In this paper, we used a model simulating pore throat. At the left side of pore throat, we add pressure gradually and make steady flow. In the gas bubble through simulation, to reduce simulation costs we use a part of the pore throat model including throat point and adopt cyclical boundary. An oil droplet or a gas bubble flow left to right and through pore throat. We observe the pressure disturbance at the midpoint of pore throat. Physical parameters are shown in Table 1.

Table 1 Parameters

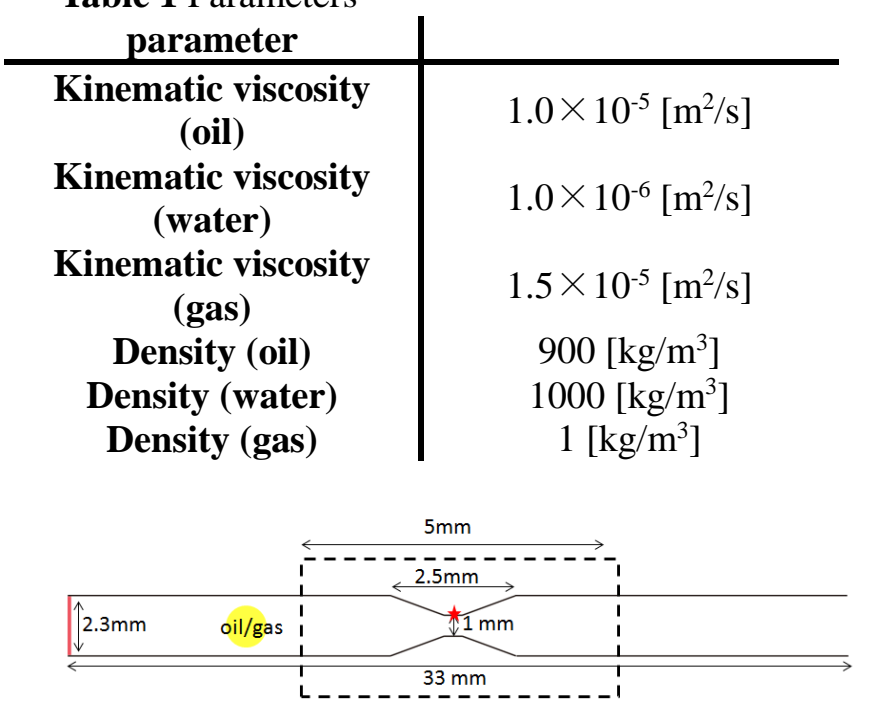

Figure 3 simulation model (fluid flow)

After observation of the pressure disturbance, we propagate seismic wave using Green's function. In the assumption isotropic homogeneous medium, we set pore throats at depth $1000 \mathrm{~m}$ and $11 \times 11$ geophones at surface each $100 \mathrm{~m}$ grid. Using the stress perturbation obtained by LBM, vibration was given at $5 \mathrm{~m}$ intervals on the plane shown in red in Figure 4 to make it flow region. The size of the oil droplets was randomly selected from the six sizes 
shown in Figure 5. It was assumed that they passed through the pore throat at an interval according to the gamma distribution of 0.1 seconds on average (Figure 6). In addition, each vibration point is passed through an oil droplet using a different random number.

In addition, we assume noisy environment and add white noise to all geophones. The noise amplitude is set to $0.1 \mathrm{~S} / \mathrm{N}$ ratio. We showed the change of the fluid flow region by changing the region of vibration. At the first stage, we gave vibration only in the blue area in Figure 7. After that, we also vibrated the green / red area as time proceeds.

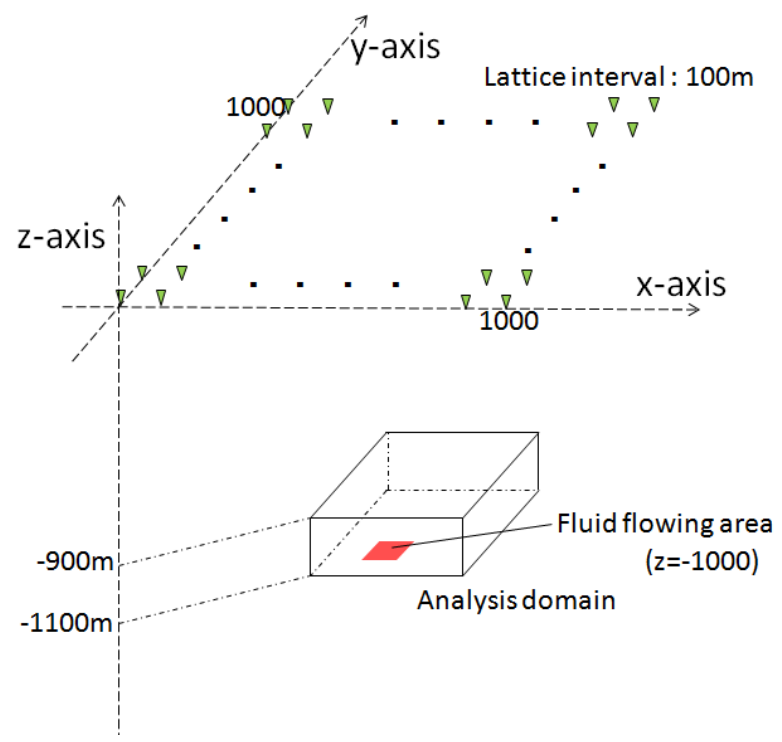

Figure 4 simulation model (wave propagation and estimation)

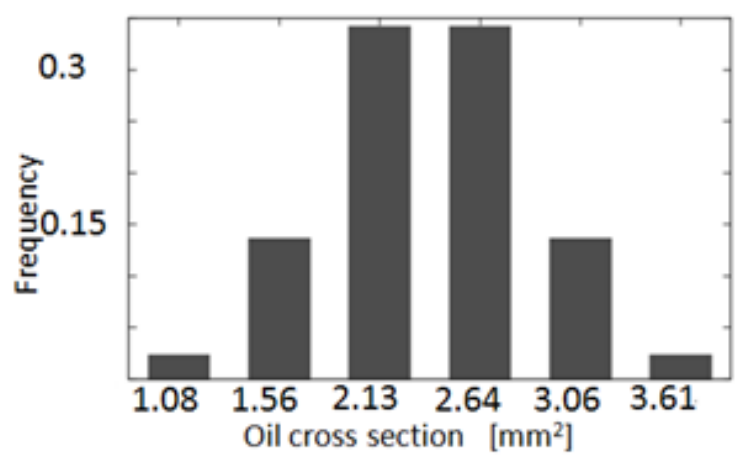

Figure 5 oil cross section and frequency

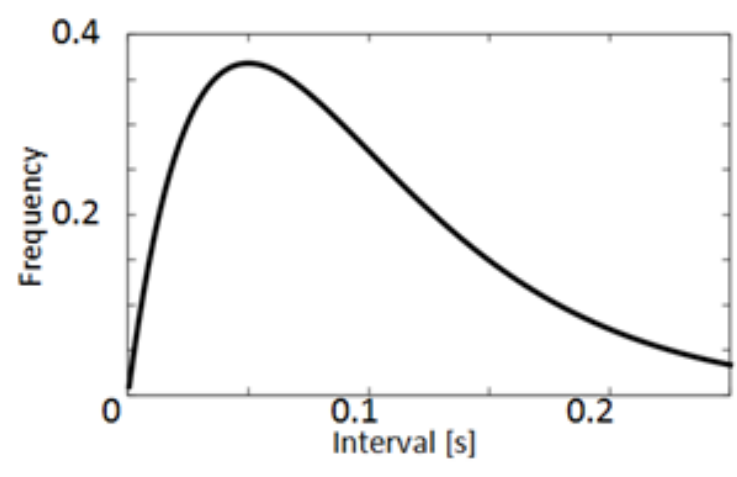

Figure 6 oil droplet pass through interval (gauss distribution, average $=0.1 \mathrm{sec}$ )

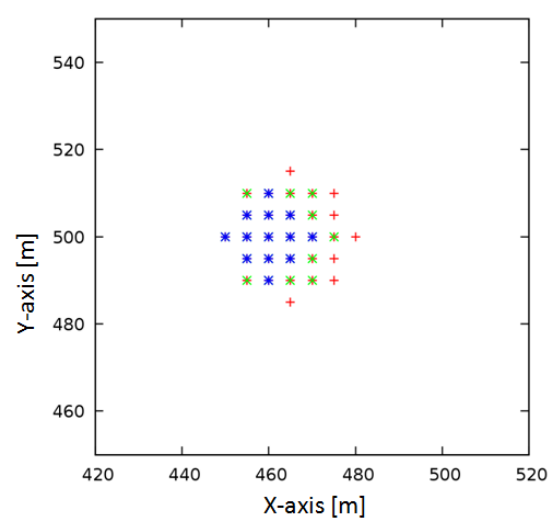

Figure 7 fluid flowing area. We change area blue to red with time passing.

\section{RESULT}

Stress disturbance was observed on the wall surface of the shrinkage portion when bubbles and oil droplets passed. Each normal stress change was $31 \mathrm{~Pa}$ when oil droplets passed and $252 \mathrm{~Pa}$ when bubbles passed (Figure 8 and 9). A repetitive oscillation is observed in the stress disturbance in bubble passage because the cyclic boundary condition is used and bubbles pass through multiple times. From these results, it was shown that the vibration generated by the properties of the flowing fluid changes even with the same pore throat.

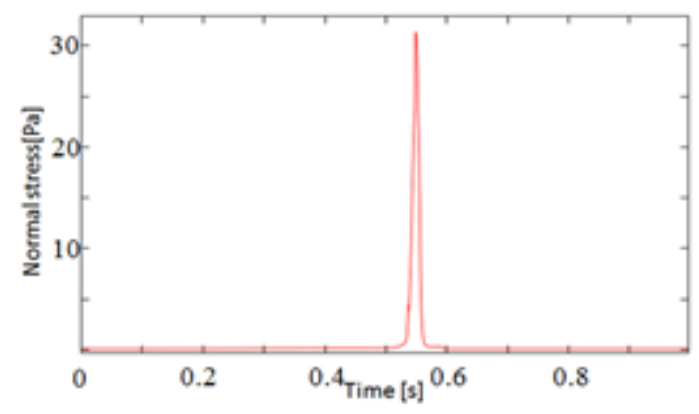

Figure 8 normal stress changes when oil droplet through pore throat 


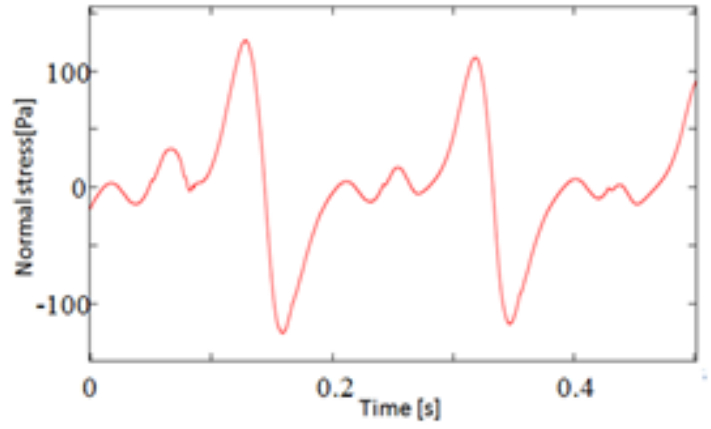

Figure 9 normal stress changes when gas bubble through pore throat
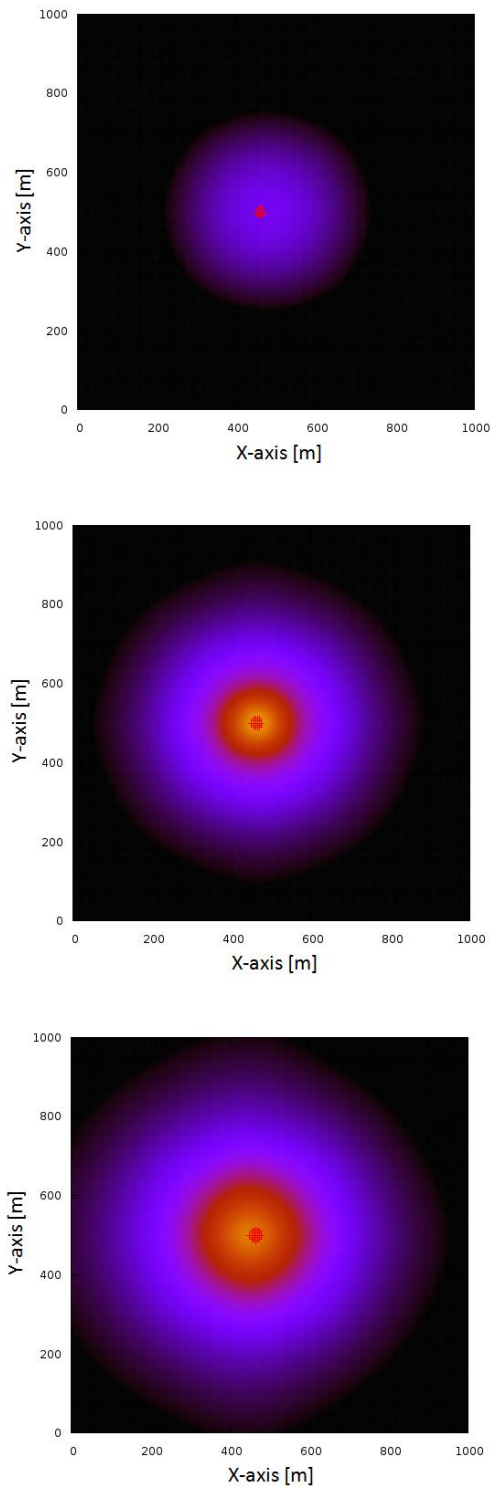

Figure 10 estimation result

\section{DISCUSSION AND CONCLUSION}

In this study, we worked on elucidation of two issues: i) what kind of stress disturbance is induced by fluid flow, ii) estimation possibility of internal fluid properties using recorded seismic waveforms. We conducted water-oil and gas-liquid two-phase flow simulation using LBM. As a result, stress disturbance was measured when oil droplets and air bubbles passed through. In addition, the stress disturbance value was 9 times as great as the difference in internal fluid despite using same pore throat. From this result, it is suggested that the difference of internal fluid can be estimated by observing the seismic wave generated by the fluid flow. In addition, we conducted a simulation under the changing situation of the fluid flow region. Changes in the fluid flow area could be observed by changing the data section used for correlation.

As a future prospect, we are paying attention to the reservoir pressure reduction phenomenon associated with oil production. It is generally known that gases dissolved in petroleum are generated as bubbles as the pressure decreasing. Using the increase in stress disturbance value due to gas bubble contamination, we are planning to investigate whether PSET can estimate reservoir pressure change.

\section{REFERENCES}

1) Ted Shuck, David E. Diller "Separating weak surface microseismic events from noise" 86th SEG Conference expanded abstract, 2016

2) Erokhin, G. N., et al. "Small microseismic surface acquisition system case study." 76th EAGE Conference and Exhibition-Workshops. 2014.

3) Rothman, Daniel H., and Jeffrey M. Keller. "Immiscible cellular-automaton fluids." Journal of Statistical Physics 52.3-4 (1988): 1119-1127.

4) Inamuro, T., et al. "A lattice Boltzmann method for incompressible two-phase flows with large density differences." Journal of Computational Physics 198.2 (2004): 628-644.

5) Swift, Michael R., W. R. Osborn, and J. M. Yeomans. "Lattice Boltzmann simulation of nonideal fluids." Physical Review Letters 75.5 (1995): 830. 